\title{
Twenty Years of Airborne Water Vapor and Total Water Measurements of a Diode Laser Based Photoacoustic Instruments
}

\author{
Miklós Szakáll ${ }^{1,2 *}$, Árpád Mohácsi ${ }^{1}$, Dávid Tátrai ${ }^{1 \dagger}$, Anna Szabó ${ }^{1}$, Helga Huszár ${ }^{1}$, \\ Tibor Ajtai ${ }^{1}$, Gábor Szabó ${ }^{1}$ and Zoltán Bozóki ${ }^{1}$ \\ ${ }^{1}$ Department of Optics and Quantum Electronics, University of Szeged, Szeged, Hungary, ${ }^{2}$ Institute for Atmospheric Physics, \\ Johannes Gutenberg University of Mainz, Mainz, Germany
}

OPEN ACCESS

Edited by:

Yufei Ma,

Harbin Institute of Technology, China

Reviewed by:

Xiaoming Gao,

Chinese Academy of Sciences

(CAS), China

Ying $\mathrm{He}$,

Harbin Institute of Technology, China

Zongliang Wang,

Liaocheng University, China

${ }^{*}$ Correspondence:

Miklós Szakál

szakall@physx.u-szeged.hu

tPresent address:

Dávid Tátrai,

Furukawa Electric Institute of

Technology, Budapest, Hungary

Specialty section:

This article was submitted to

Optics and Photonics,

a section of the journal

Frontiers in Physics

Received: 27 March 2020

Accepted: 07 August 2020

Published: 01 October 2020

Citation:

Szakáll M, Mohácsi Á, Tátrai D, Szabó A, Huszár H, Ajtai T, Szabó G and Bozóki Z (2020) Twenty Years of Airborne Water Vapor and Total Water

Measurements of a Diode Laser

Based Photoacoustic Instruments.

Front. Phys. 8:384

doi: 10.3389/fphy.2020.00384
We present relevant issues collected over 20 years of development and operation of an airborne water vapor/total water detector based on photoacoustic spectroscopy. The WaSul-Hygro instrument possesses high selectivity, short response time, and wide dynamic range, which are key requirements against trace gas measurement systems for applications in atmospheric sciences. Besides the major properties of WaSul-Hygro, we discuss our efforts to develop a robust instrument that has proven its long-term reliability over the last 15 years operating onboard a commercial aircraft within the framework of the CARIBIC project.

Keywords: photoacoustics, diode laser, airborne measurements, humidity, instrument development

\section{INTRODUCTION}

Water vapor is the most important atmospheric constituent that has a large impact on the Earth's radiation budget. Besides the direct radiative forcing of water vapor, its indirect effect by generating cloud droplets plays also a crucial role in the climate. Hence, the precise and regular characterization of its abundance in the atmosphere are of key importance especially in the changing climate system. In the upper troposphere/lower stratosphere (UTLS) region of the atmosphere, water vapor drives the generation of pure ice (cirrus) clouds through homogeneous or heterogeneous freezing processes and the growth of the cloud ice particles by deposition. The radiative effect of cirrus clouds is still not understood; some studies show their cooling, whereas others their warming effect, depending on the representation of cloud optical thickness and ice particle size and concentration. Within the framework of the European CARIBIC project $[1,2]$ (Civil Aircraft for the Regular Investigation of the Atmosphere Based on an Instrument Container), atmospheric water vapor and total water (i.e., the sum of water vapor and cloud water/ice) concentration have been measured onboard a commercial aircraft regularly in the UTLS region (at 10- to $12-\mathrm{km}$ altitude) since 2005 utilizing a photoacoustic (PA) method-based instrument developed in our laboratory.

The airborne PA water vapor measuring instrument (called WaSul-Hygro) is based on a telecommuncation-type near-infrared (NIR) diode laser. Furthermore, in order to ensure the requirements for the simultaneous measurement of total water and water vapor, WaSul-Hygro possesses a dual-chamber PA setup optimized for low-temperature and low-pressure conditions. This type of operation is facilitated by a special environmental air inlet system attached below the aircraft containing both a sideward- and a forward-facing inlet, which sample water vapor 
and total water, respectively [3]. Each inlet is individually connected to one of the two PA cells, thus establishing separate measurement of water vapor and total water. The electropolished stainless-steel sampling lines (heated to $60^{\circ} \mathrm{C}$ ) ensure that the entire water content of the sampled particles evaporates before entering the WaSul-Hygro instrument where, therefore, gas-phase measurements are performed. To permanently maintain reliable fully automated long-term operation onboard a commercial aircraft, where daily maintenance is not possible, hardware components of WaSul-Hygro were carefully matched, and various self-checking and self-correcting procedures were built into its operational software.

The aim of this article is to provide a comprehensive summary of relevant scientific and technical issues collected during the last two decades of development and operation of WaSul-Hygro. In particular, the advantages of the PA method for high-precision gas concentration measurements and the main characteristics of the airborne PA water vapor sensor, such as mechanical and optical setup, optimal laser modulation parameters, pressure dependent sensitivities, etc., will be discussed. After that, we introduce the operation of the airborne water vapor detector, WaSul-Hygro, and present measurement data collected onboard a commercial aircraft.

\section{ADVANTAGES OF THE PA DETECTION METHOD}

PA spectroscopy is a light absorption spectroscopic method; i.e., the concentration of the measurable gas is determined by measuring the light intensity absorbed during penetrating through this gas [4]. Thus, the main components of a PA instrument are a light source; a chamber containing the gas, the concentration of which has to be measured; and a detector that measures the adsorbed light intensity (Figure 1).

The light source of WaSul-Hygro is a distributed feedback (DFB) diode laser operating at $1,371 \mathrm{~nm}$. These telecommunication-type NIR room temperature diode lasers are robust, long-living, and reliable and can be operated completely automatically, which are key properties for instruments in airborne applications. Furthermore, DFB lasers emit light in a very narrow spectral wavelength range that can continuously be tuned over 2 to $3 \mathrm{~nm}$ by changing the lasers' temperature with a typical tuning rate of $0.1 \mathrm{~nm} / \mathrm{K}$. Disadvantages of DFB lasers are their relatively low light power (typically a few tens of $\mathrm{mW}$ ) and that they are operating at wavelengths of overtone or combination bands of molecular vibrational transitions that have relatively small absorption strengths. This limits the achievable minimum detectable concentration (MDC) in spectroscopic applications to the ppm to ppb level, which is nevertheless sufficient for airborne water vapor measurements in UTLS.

The PA chamber through which the ambient air was sucked through was constructed to suppress outside noise originated mainly from the cruising air flow and from the aircraft, and to amplify the generated sound waves. The PA cell design applied in WaSul-Hygro was a longitudinal differential PA cell. In this construction, two resonators with lengths of $\sim 4 \mathrm{~cm}$ and corresponding $4,800-\mathrm{Hz}$ resonance frequency are placed in parallel: the light propagates through only one of the resonators, in which, therefore, the PA signal is generated. Subtracting the signal measured in the non-illuminated idle resonator from the PA signal, high-efficient noise suppression is performed. Four cavities (two on each side of the resonators and two at the gas sampling inlet and outlet) served for background noise reduction. Each PA cell built in WaSul-Hygro has a volume of $\sim 15 \mathrm{~cm}^{3}$ and can be operated at a volumetric flow rate of $\sim 15 \mathrm{~cm}^{3} / \mathrm{s}$.

The PA effect bears on the phenomenon that sound wave is generated in a medium absorbing light of modulated intensity or wavelength. This sound is generally measured in PA spectroscopic systems, hence, in WaSul-Hygro, too, by a microphone.

\section{FEATURES OF AIRBORNE PA WATER VAPOR DETECTOR WASUL-HYGRO}

As highlighted above, PA spectroscopy possesses a set of features making it an ideal tool for airborne applications [5]. The main requirements against instruments measuring trace gas concentration onboard an aircraft are high selectivity, short

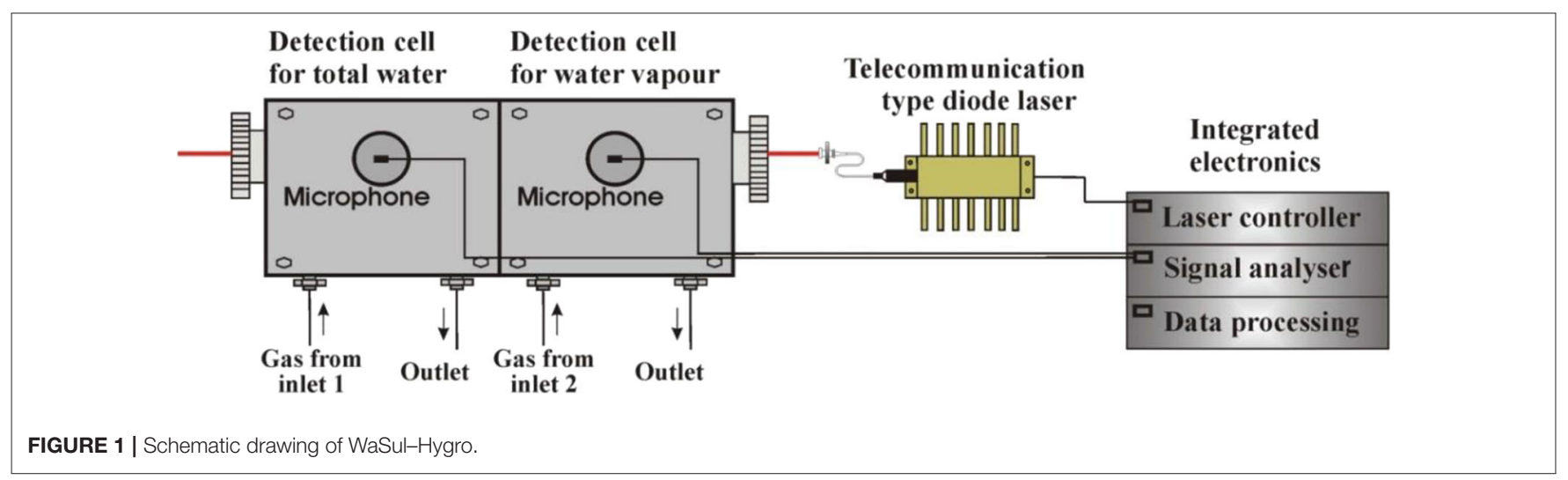


response time, and wide dynamic range of measurable gas concentration. The PA spectroscopic measurement technique allows direct measurement of analyte concentration in the gas phase without applying any analyte conversion process. This high selectivity was demonstrated by intercomparing the first-generation WaSul-Hygro system with a dew point hygrometer under simulated atmospheric conditions in the AIDA chamber [6]. In polar stratospheric cloud simulation experiments, water vapor concentration was measured in the presence of nitric acid vapor. Because nitric acid does not have optical absorption in the applied wavelength range optimized for water vapor measurements, the PA system measured water vapor concentration without any interference with nitric acid. In contrast, the dew point hygrometer was influenced by nitric acid, because it was precipitated onto the chilled mirror surface and falsified its measurement. In a more recent airborne intercomparison campaign [7], we proved that the repeatability $(0.5 \mathrm{ppmV}$ or $0.5 \%)$, the accuracy ( $5 \%$ of the actual reading), and the response time (2s) of a WaSul-Hygro system dedicated for water vapor mixing ratio qualify it for water vapor measurements in UT. Furthermore, Quartz-Enhanced Photoacoustic Spectroscopy (QEPAS), a PA-based spectroscopy development of recent years, seems to be a very promising candidate for further enhancing the sensitivity of PA sensors and to widen their field of application by measuring besides water vapor, e.g., acetylene, carbon monoxide, etc. [8-10].

The small volume $\left(15 \mathrm{~cm}^{3}\right)$ and the possible high operational volumetric flow rate $\left(15 \mathrm{~cm}^{3} / \mathrm{s}\right)$ ensure a fast $(\sim 1 \mathrm{~s})$ air sample exchange in the PA cell. Taking the cruising speed of the aircraft $(250 \mathrm{~m} / \mathrm{s})$ into account, this response time enables a spatial resolution of $250 \mathrm{~m}$ for water vapor measurements with WaSulHygro. Allan variance analysis of the averaging time of the measured PA signal resulted in an optimal averaging time of $2 \mathrm{~s}$. However, because of supplementary self-checking procedures, the real measurement time is increased to $2.7 \mathrm{~s}$.

In UTLS applications of WaSul-Hygro, the water vapor concentration to be measured ranged from 4 ppm up to 40,000 ppm (considering also total water concentrations). The lowest end of the measurement range corresponds to the self-noise of the detector, which is $N=150 \mathrm{nV} / \sqrt{ } \mathrm{Hz}$. For the PA cells and the diode laser built in WaSul-Hygro, a minimum detectable optical absorption coefficient of $\alpha_{\min }=5 \times 10^{-7} \mathrm{~cm}^{-1}$ can be calculated. The higher end of the measurement range corresponds to a power loss of $\Delta P / P_{0} \approx 60 \%$ in the PA cell. Because the optical path length through the PA cell is about $10 \mathrm{~cm}$, the maximum detectable optical absorption coefficient is $\alpha_{\max } \approx 0.1 \mathrm{~cm}^{-1}$. Thus, a dynamic range of $\alpha_{\max } / \alpha_{\min } \approx 2 \times 10^{5}$ for WaSul-Hygro is estimated. Hence, the minimum and maximum detectable water vapor concentrations are 0.75 and 150,000 ppm, respectively. Unfortunately, the intrinsically wide dynamic range of WaSul-Hygro was restricted by the limited dynamic range of the applied analog-to-digital converter, which necessitated the application of a sensitivity switching at high concentrations of the measured analyte as follows. When the PA signal is above a predefined limit, the system switches automatically to a less sensitive operation mode. On the contrary, when the PA signal becomes too low, the sensitivity is switched back, but with a hysteresis between the two switching points. In WaSul-Hygro, sensitivity switching is realized by detaching the laser modulation frequency from the acoustic resonance frequency of the PA cell, which procedure can be executed very quickly.

\section{OPERATION OF WASUL-HYGRO}

WaSul-Hygro was constructed for simultaneous measurement of water vapor and total water. Because the water vapor concentration in UTLS is low, and the optical path length in the PA cell is short, the decrease in light intensity after the PA cell due to absorption is negligible. Therefore, we placed a second PA cell adjacent the first one in a way that the outlet window of PA cell 1 became the inlet window of PA cell 2 (see Figure 1). In this way, the same laser is used for PA signal generation in both PA cells. The air sampling method described above together with the two-cell design enabled the high accuracy detection of water vapor and cloud water. In clear sky conditions, water molecules are present only in vapor form, and the concentrations measured by the two PA cells are identical. When the aircraft is flying in clouds, the difference measured in the two distinct PA cells quantifies the amount of cloud particles. Furthermore, the PA cells of WaSul-Hygro are mounted onto a common heating plate, which keeps their temperatures at the same constant value. Any change in the temperature of the PA cells would falsify the concentration measurement, because both the responsivity of the microphone and the resonance frequency of the PA cells depend on the temperature [11].

To achieve the ultimate goal of high-accuracy water vapor and total water measurements onboard an aircraft in the UTLS, the MDC of WaSul-Hygro had to be in the ppb regime. This was realized by maximizing the signal-to-noise and signal-tobackground ratios.

To obtain as large PA signal as possible, a diode laser was employed, which operates at a wavelength where water vapor has a strong absorption line $(1,371 \mathrm{~nm})$. Furthermore, because this absorption line is narrow, wavelength modulation was applied. In this modulation scheme, the diode laser wavelength is tuned periodically on and off the water vapor absorption line at a frequency that equals the resonance frequency of the PA cells (i.e., $4,800 \mathrm{~Hz}$ ). The absorption linewidth at atmospheric pressure is $0.03 \mathrm{~nm}$, whereas the wavelength tuning rate of the WaSul-Hygro diode laser is $0.005 \mathrm{~nm} / \mathrm{mA}$. Hence, the maximal modulation of the absorbed line at atmospheric pressure is achieved when $I_{\mathrm{AC}} \approx 6 \mathrm{~mA}$ laser current modulation amplitude is used. However, in airborne applications, the wavelength modulation has to be optimized for different pressure levels [12], because the total pressure of the gas sample varies at different altitudes. For example, the width of the water vapor absorption line decreases to $0.008 \mathrm{~nm}$ when the pressure drops to $190 \mathrm{hPa}$ (i.e., at the aircraft's cruising level); therefore, the optimum $I_{\mathrm{AC}}$ changes to $1.5 \mathrm{~mA}$. Because the water vapor concentration has its lowest value at about $300 \mathrm{hPa}$, we optimized the wavelength modulation parameters in WaSul-Hygro to ensure maximum sensitivity at this pressure level [13]. In 


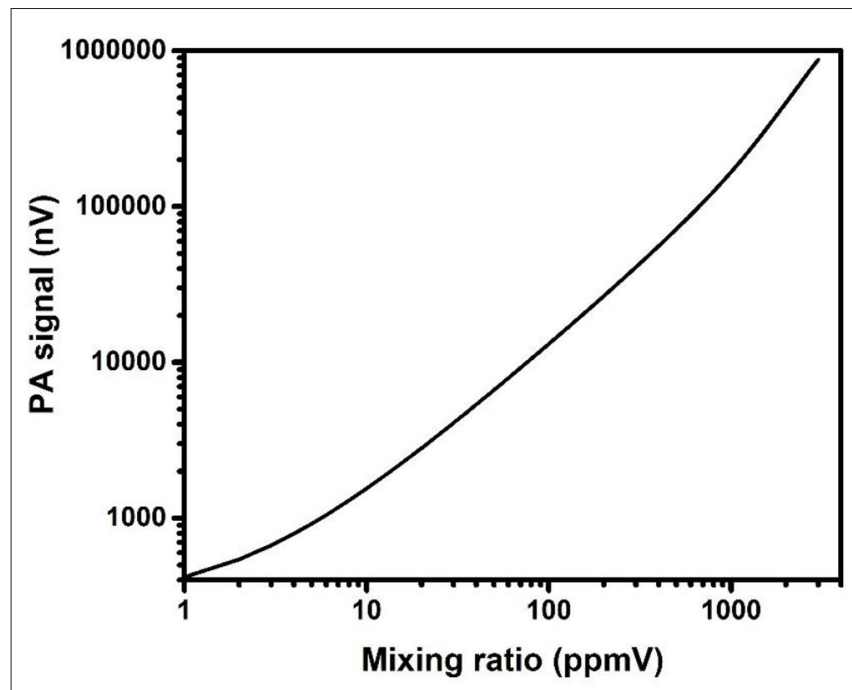

FIGURE 2 | Calibration curve for the water vapor concentration measurement PA cell of WaSul-Hygro performed under laboratory conditions.

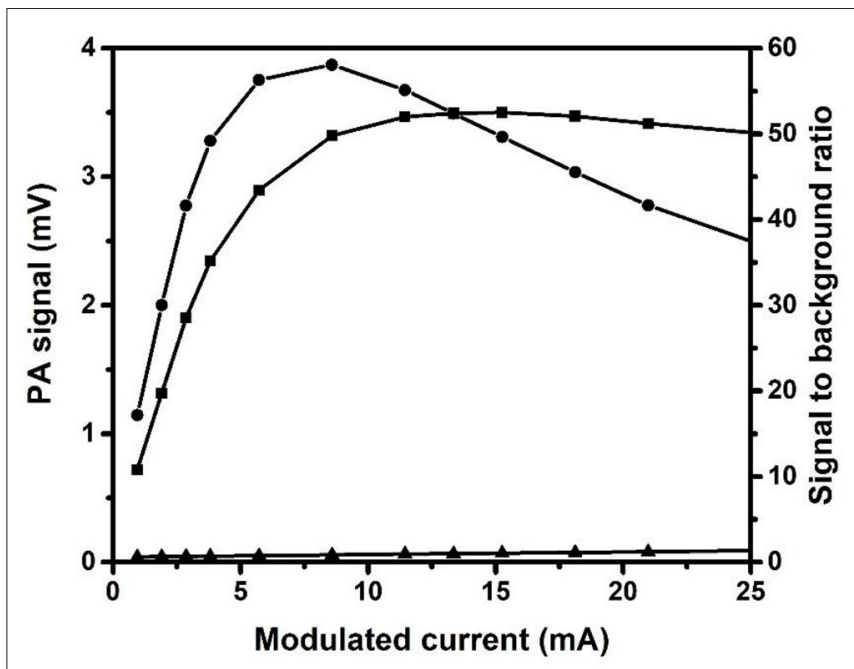

FIGURE 3 | Modulation amplitude dependence of the PA signal (marked with squares), the PA background signal (marked with triangles), and the ratio of these two signals (marked with circles). The measurements were carried out at atmospheric pressure in laboratory using a diode laser operating at the water vapor absorption line at $1,371 \mathrm{~nm}$.

enhanced. Unfortunately, modulating the laser current causes not only wavelength modulation, but also the light intensity is modulated as well. Because of this residual amplitude modulation effect, background PA signals cannot be entirely eliminated even in case of wavelength modulation. Furthermore, the larger the $I_{\mathrm{AC}}$ is, the bigger the background PA signal will be (Figure 3). This necessitated the optimization of the wavelength modulation in WaSul-Hygro in order to maximize not only the signalto-noise ratio but also the signal-to-background ratio. In the optimization procedure, which was carried out under laboratory conditions prior to the installation into the aircraft, the maximum laser driving current $I_{\max }$ was kept constant, and the laser temperature was adjusted to compensate for the laser wavelength variation stemming from the variation of $I_{A C}$.

The diode laser utilized in WaSul-Hygro showed an aging effect, i.e., its wavelength shifted during its long-term operation, resulting in a detuning from the peak of the water vapor absorption line. This effect appeared as an apparent decrease in the PA signal, which was more pronounced the lower the pressure was. Therefore, a wavelength stabilization (linelocking) method was developed and implemented in WaSulHygro [13, 15]. For the line-locking, stable high-concentration water vapor is needed in the PA cell; therefore, a third PA cell was built in series with the two measurement cells. This linelocking PA cell was sealed and contained high-concentration water vapor from atmospheric air in the ground. We note that because of recent technological development of diode lasers, they do not show any aging effect, and in a next generation of WaSul-Hygro, the use of the line-locking PA cell can be abandoned.

WaSul-Hygro was calibrated after the assembly of the instrument and was validated regularly during the operation 
period. The early version of WaSul-Hygro showed a considerable $(\sim 15 \%)$ sensitivity change during its long-term operation, which issue was eliminated in further generations of the instrument. The calibration was carried out for both PA cells following a complex procedure over a wide water vapor concentration and

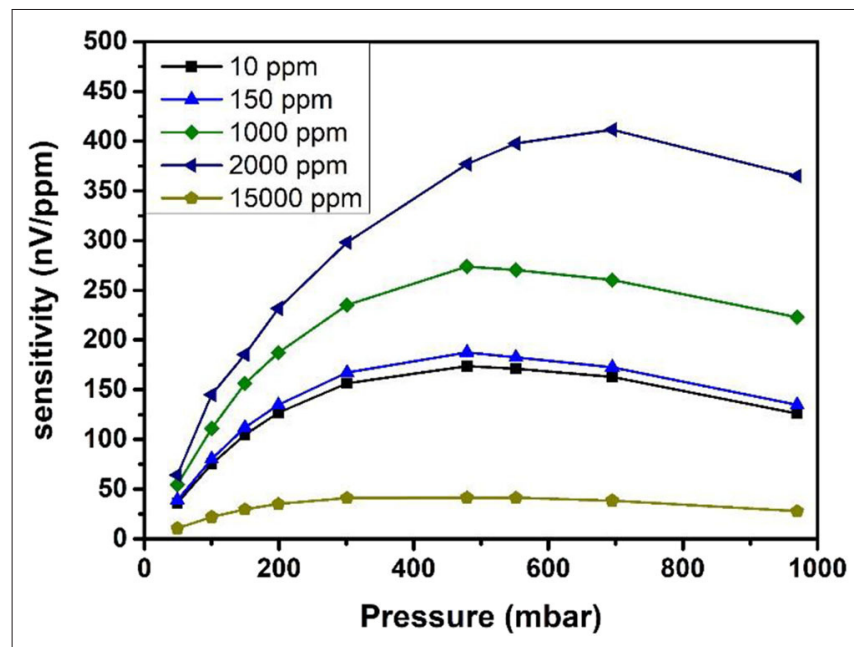

FIGURE 4 | Sensitivity dependence for the water vapor measurement PA cell of WaSul-Hygro on absolute sample air pressure and on the water vapor concentration measured during calibration under laboratory conditions. pressure range necessary for airborne applications. Furthermore, the calibration was conducted for both sensitivity switching settings. The calibration started by cooling a humidity generator developed in our laboratory to about $-120^{\circ} \mathrm{C}$ by means of liquid nitrogen and letting it to warm up gradually to room temperature. The water vapor concentration of the calibrator gas was determined using the Clausius-Clapeyron equation from the continuously measured humidity generator temperature. In case of slowly heating up of the humidity generator, it was possible to obtain calibration curves for each pressure level in a single calibration run. With the latest version of our humidity generator, we were able to calibrate WaSul-Hygro quasi-simultaneously at 10 different pressure levels. The water vapor and total water concentrations were continuously calculated in-line from the measured PA signals in the two PA cells. In the early version of WaSul-Hygro, a systematic error in the concentration determination was generated, because the calculation was based on the erroneous presumption that the system's sensitivity is independent on the water vapor concentration. In the second generation of WaSul-Hygro, we eliminated this issue by introducing a pressure-dependent sensitivity factor, which also has concentration dependence (Figure 4). The applied concentration determination procedure meets the ultimate requirement that the water vapor concentration deviation between the instrument's reading and the actual value is within the noise level of the measurement. Furthermore, the concentration determination is executed within $100 \mathrm{~ms}$ and thus does not consume useful time from PA signal measurements.

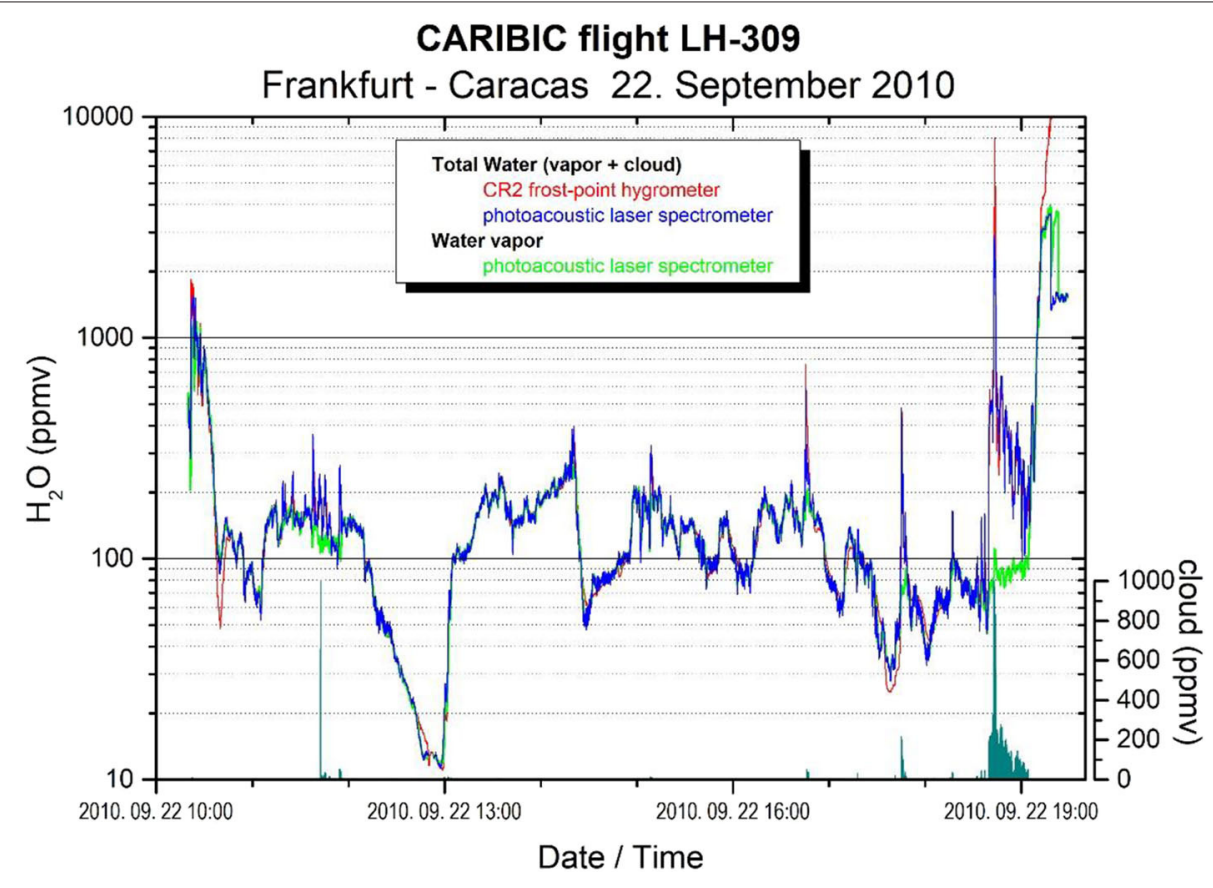

FIGURE 5 | Water vapor and total water concentrations measured by WaSul-Hydro in a CARIBIC flight from Frankfurt (Germany) to Caracas (Venezuela). The concentration of cloud water/ice was calculated as the difference of water vapor and total water (by considering an enhancement factor of 3 to 4 due to the CARIBIC inlet). The total water was also measured by a one-channel frost point hygrometer (CR-2). 


\section{SUMMARY}

The WaSul-Hygro system has been operating onboard a commercial aircraft within the CARIBIC project since 2005. However, detailed and comprehensive analysis of airborne data collected by WaSul-Hygro has not yet been carried out; an example of valuable measurement results is shown in Figure 5. These data were recorded during a flight from Frankfurt (Germany) to Caracas (Venezuela). The total water measurements of WaSul-Hygro were compared to the measurements of a commercially available frost point hygrometer (CR-2, Buck Research Instruments, LLC). The detailed analysis of the data is also necessary in order to ascertain the reason of sensitivity reduction of WaSul-Hygro onboard the CARIBIC aircraft and to collect information about the long-term performance of the system components and the measurement methodology. Nevertheless, within the last two decades, a series of self-checking and self-correcting algorithms were developed and implemented in the new version of WaSul-Hygro, and a bunch of novel hardware components was tested and carefully selected in order to optimize the operation of the instrument onboard an aircraft. The continuous

\section{REFERENCES}

1. Zahn A, Christner E, van Velthoven PFJ, Rauthe-Schöch A, Brenninkmeijer CAM. Processes controlling water vapor in the upper troposphere/lowermost stratosphere: An analysis of 8 years of monthly measurements by the IAGOSCARIBIC observatory-CARIBIC $\mathrm{H}_{2} \mathrm{O}$ in the UT/LMS. J Geophys Res. (2014) 119:11.505-25. doi: 10.1002/2014JD021687

2. Dyroff C, Zahn A, Christner E, Forbes R, Tompkins AM, van Velthoven PFJ. Comparison of ECMWF analysis and forecast humidity data with CARIBIC upper troposphere and lower stratosphere observations. Q J Royal Meteorol Soc. (2015) 41:833-44. doi: 10.1002/qj.2400

3. Hermann M, Stratmann F, Wilck M, Wiedensohler A. Sampling characteristics of an aircraft-borne aerosol inlet system. $J$ Atmos Ocean Technol. (2001) 18:7-19. doi: 10.1175/15200426(2001)018<0007:SCOAAB >2.0.CO;2

4. Bozóki Z, Mohácsi Á, Szabó G, Bor Z, Erdélyi M, Chen W. Near infrared diode laser based spectroscopic detection of ammonia: a comparative study of photoacoustic and direct optical absorption methods. Appl Spectrosc. (2002) 6:715-9. doi: 10.1366/000370202760077658

5. Bozóki Z, Pogány A, Szabó G. Photoacoustic instruments for practical applications: present potentials and future challenges. Appl Spectrosc Rev. (2011) 6:1-37. doi: 10.1080/05704928.2010.520178

6. Szakáll M, Bozóki Z, Krämer M, Spelten N, Möhler O, Schurath U. Evaluation of a photoacoustic detector for water vapor measurements under simulated tropospheric/lower stratospheric conditions. Environ Sci Technol. (2001) 35:4881-5. doi: 10.1021/es015564x

7. Tátrai D, Bozóki Z, Smit H, Rolf C, Spelten N, Krämer M. Dualchannel photoacoustic hygrometer for airborne measurements: background calibration laboratory and in-flight intercomparison tests. Atmos Meas Tech. (2015) 8:33-42. doi: 10.5194/amt-8-33-2015

8. Ma Y, Qiao S, He Y, Li Y, Zhang Z, Yu X. Highly sensitive acetylene detection based on multi-pass retro-reflection-cavity-enhanced photoacoustic spectroscopy and a fiber amplified diode laser. Opt Express. (2019) 7:1416372. doi: 10.1364/OE.27.014163

9. Li Y, Wang R, Tittel FK, Ma Y. Sensitive methane detection based on quartz-enhanced photoacoustic spectroscopy with a high-power development of WaSul-Hygro ensured its robustness and long-term reliable operation even under harsh conditions in the UTLS.

\section{DATA AVAILABILITY STATEMENT}

The raw data supporting the conclusions of this article will be made available by the authors, without undue reservation.

\section{AUTHOR CONTRIBUTIONS}

MS, ÁM, DT, AS, HH, TA, GS, and ZB have been participated in the twenty years of development work related to the presented system. All authors contributed to the article and approved the submitted version.

\section{ACKNOWLEDGMENTS}

The preparation of this paper is supported by the TUDFO/471381/2019-ITM FIKP program, the Ginop-2.3.2-15-2016-00036 project and by the János Bolyai Research Scholarship of the Hungarian Academy of Sciences received by TA.

diode laser and wavelet filtering. Opt Lasers Eng. (2020) 32:106155. doi: 10.1016/j.optlaseng.2020.106155

10. Ma Y, Qiao S, Patimisco P, Sampaolo A, Wang Y, Tittel FK. In plane quartzenhanced photoacoustic spectroscopy. Appl Phys Lett. (2020) 16:061101. doi: $10.1063 / 1.5142330$

11. Szakáll M, Csikós J, Bozóki Z, Szabó G. On the temperature dependent characteristics of a photoacoustic water vapor detector for airborne applications. Infrared Phys Technol. (2007) 1:113-21. doi: 10.1016/j.infrared.2007.04.001

12. Szakáll M, Huszár H, Bozóki Z, Szabó G. On the pressure dependent sensitivity of a photoacoustic water vapor detector using active laser modulation control. Infrared Phys Technol. (2006) 8:192-201. doi: 10.1016/j.infrared.2006.01.002

13. Szakáll M, Bozóki Z, Mohácsi Á, Varga A, Szabó G. Diode laser based photoacoustic water vapor detection system for atmospheric research. Appl Spectrosc. (2004) 8:792-8. doi: 10.1366/00037020413 89373

14. Lang B, Bretegger P, Brunnhofer G, Valero JP, Schweighart S, Klug $A$, et al. Molecular relaxation effects on vibrational water vapor photoacoustic spectroscopy in air. Appl Phys B. (2020) 126:64. doi: 10.1007/s00340-020-7409-3

15. Tátrai D, Bozóki Z, Szabó G. Method for wavelength locking of tunable diode lasers based on photoacoustic spectroscopy. Opt Eng. (2013) 52:096104. doi: 10.1117/1.OE.52.9.096104

Conflict of Interest: The authors declare that the research was conducted in the absence of any commercial or financial relationships that could be construed as a potential conflict of interest.

Copyright (C) 2020 Szakáll, Mohácsi, Tátrai, Szabó, Huszár, Ajtai, Szabó and Bozóki. This is an open-access article distributed under the terms of the Creative Commons Attribution License (CC BY). The use, distribution or reproduction in other forums is permitted, provided the original author(s) and the copyright owner(s) are credited and that the original publication in this journal is cited, in accordance with accepted academic practice. No use, distribution or reproduction is permitted which does not comply with these terms. 